\title{
Glosse
}

\section{Scheidung oder Schlaganfall?}

_ Männer haben es nicht immer leicht im Leben. Ständig werden sie mit Situationen konfrontiert, die das Wesen eines Dilemmas in sich tragen, d.h. jede Entscheidung birgt das Risiko eines Irrtums, um nicht zu sagen eines Fehlers in sich. Errare virile est! Meist geht es darum, das kleinere Übel zu wählen: Mercedes oder BMW, Ehefrau oder Geliebte, Golf oder Tennis, Karriere oder Gesundheit? Jetzt hat eine aktuelle Studie einen neuen Zielkonflikt mit tragischen Folgen offenbart, dem sich letztlich kein Mann entziehen kann.

Auf der Suche nach psychosozialen Risikofaktoren für ein vaskuläres Ereignis hat sich die Wissenschaft mit der täg- lichen Arbeitszeit beschäftigt und diese in einen kausalen Zusammenhang mit der Dauer der Ehe, also der Scheidungsrate gestellt. Und dabei ergab sich interessanterweise ein geschlechtsspezifischer Unterschied: Frauen, die beruflich sehr stark engagiert sind, werden häufiger geschieden, ja es fand sich sogar eine strenge Korrelation zwischen wöchentlicher Arbeitszeit und Scheidungsrate. Doch bei Männern ist es genau umgekehrt: Wer viel und lange arbeitet, dessen Ehe ist weniger gefährdet als bei einer normalen 36-Stunden-Woche.

Man könnte also mutmaßen: Wer seine Frau kaum noch sieht, führt eine glücklichere Ehe als der, der pünktlich nach der tariflich vereinbarten Arbeitszeit zu Hause erscheint. Dass diese Studie für Ärzte höchst relevant ist, bedarf wohl keines Zweifels.

Soweit, so gut. Aber wo Licht ist, ist bekanntlich auch Schatten. Denn Männer mit langen Arbeitszeiten bekommen nach dieser Studie häufiger einen Schlaganfall, bei Frauen konnte dies so nicht dokumentiert werden. Somit muss der Mann sich nun entscheiden: Scheidung oder Schlaganfall? Also ob er einen Schlaganfall in einem ehelichen Status einem Leben ohne Schlaganfall, aber auch ohne Ehefrau vorziehen möchte? Ob aber die Arbeitsbelastung selbst oder gar die Ehe der eigentliche Auslöser des Schlaganfalls darstellt, darüber wollten die Forscher sich aus nachvollziehbaren Gründen lieber nicht äußern.

Dr. Peter Stiefelhagen

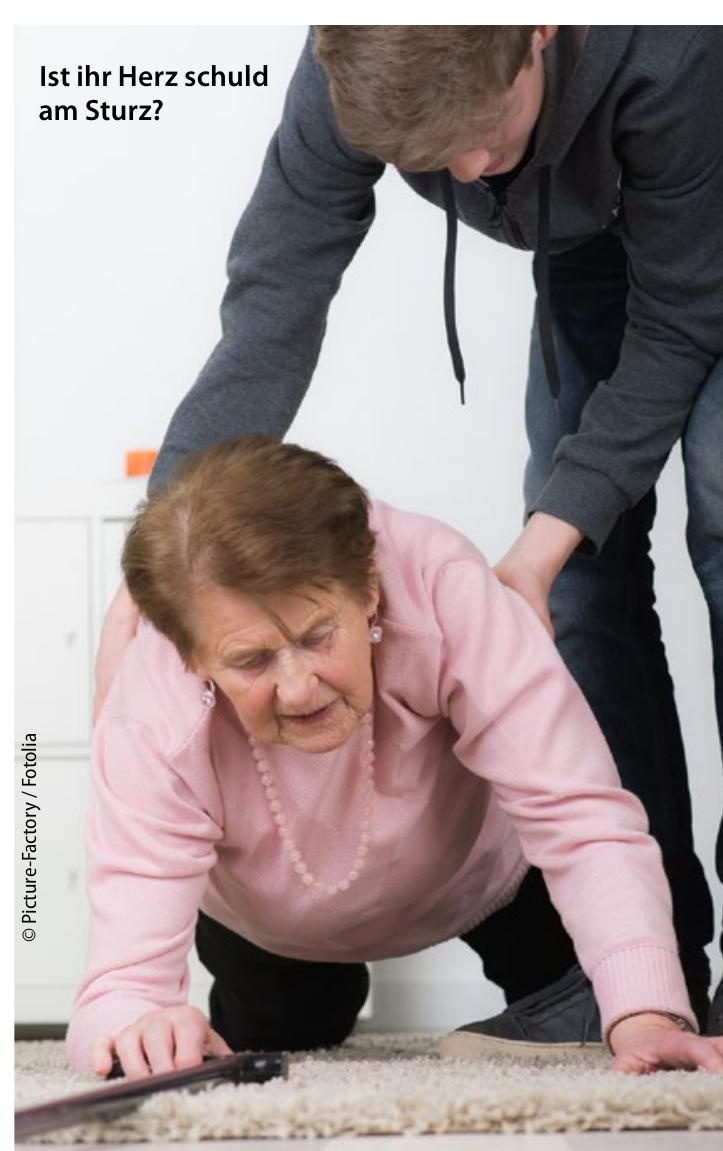

\section{Herzrhythmusstörungen}

\section{Eventrekorder klärt Sturzursachen auf}

Ungeklärte Stürze und Synkopen älterer Menschen gehen offenbar zu einem Großteil auf kardiale Arrhythmien zurück. Dies ist das Ergebnis einer Studie, in der die Probanden einen Ereignisrekorder unter der Haut getragen hatten.

Kardiale Arrhythmien werden als möglicher Sturzauslöser zu selten diagnostiziert, so die Vermutung von Gerontologen vom Trinity College in Dublin. Um ihre Hypothese zu prüfen, nahmen sie 70 Patienten (mittleres Alter 70 Jahre) in eine Studie auf. Alle hatten im vorangehenden Jahr mindestens zweimal wegen eines Sturzes eine Notaufnahme aufsuchen müssen. Um eine längerfristige Suche nach kardialen Sturzauslösern zu ermöglichen, implantierten die Ärzte den Patienten einen Ereignisrekorder subkutan in die linke Parasternalregion. Die Nachbeobachtungszeit mit Rekorder betrug im Mittel 9 Monate.

Kardiale Arrhythmien entdeckten die Geräte bei 50 Patienten (71\%), darunter Vorhofflimmern (4 Patienten), supraventrikuläre Tachykardien (12) sowie Bradykardien (34). Bei 14 Patienten (20\%) ereignete sich zeitgleich mit der Rhythmusstörung ein Sturz bzw. eine Synkope. „Jeder fünfte Studienpatient, der Stürze aus zuvor ungeklärter Ursache erlitten hatte, wies eine Arrhythmie auf, mit der sich die Stürze erklären ließen“, so die Autoren. Generell gehe die Diagnose einer Arrhythmie mit einem höheren Risiko für weitere Stürze einher.
- Bhangu J et al. Heart 2016;102:681-686

- rb 